\title{
Patrones de emergencia de Odonata (Insecta) en un hábitat lótico de Cuba oriental
}

\author{
Adrian Trapero-Quintana \& Bernardo Reyes-Tur \\ Departamento de Biología, Universidad de Oriente. Ave. Patricio Lumumba s/n. Santiago de Cuba 90500, Cuba; \\ trapero76@gmail.com, breyestur@gmail.com
}

\author{
Recibido 06-VII-2016. Corregido 16-I-2017. Aceptado 07-II-20176.
}

\begin{abstract}
Emergence patterns of Odonata (Insecta) from a lotic habitat in Eastern Cuba. The emergence patterns of tropical odonates (dragonflies and damselflies) are scarcely known. We studied the emergence patterns of odonates in a freshwater lotic system in Giro, Northern Santiago de Cuba, between January and December 2008. We visited the locality between 09:00 and 14:00, on a weekly basis, and collected exuviae from a fixed section $\left(8 \times 1 \mathrm{~m}^{2}\right)$ offshore, along the riparian vegetation. We collected data on species composition and, for each species, abundance, relative biomass and emergence pattern. We collected 443 exuviae belonging to 22 species: seven Zygoptera and 15 Anisoptera. Half of the annual Odonata emergence took place in the dry season (December to March) with the highest value in February (25\%). For species for which we found seven or more exuviae per month, Enallagma coecum and Macrothemis celeno tended to be a synchronal emergence. We also found temporal segregation of the emergence pattern between M. celeno and Protoneura capillaris, Neoneura maria, Progomphus integer and Scapanea frontalis. These differences were probably related to the highest annual fluctuations of temperature, relative humidity and number of rainy days per month. We concluded that there is an asynchrony and heterogeneity in Odonata emergence times in the studied freshwater lotic system. Rev. Biol. Trop. 65 (2): 807-818. Epub 2017 June 01.
\end{abstract}

Key words: Odonata, exuviae, emergence patterns, climatic factors, Cuba.

Los odonatos son reconocidos por su papel ecológico como depredadores y presas en la estructura trófica de las comunidades acuáticas (Watson, 1982; Corbet, 2004). Sus larvas son generalmente acuáticas, excepto algunas que se desarrollan en ambientes semiacuáticos o terrestres. Una vez concluido el ciclo larval tiene lugar la emergencia, que incluye el desplazamiento de las larvas al medio aéreo. Posteriormente, a partir de procesos hormonales, se separa el dermatoesqueleto (exuvia), transformándose la larva en un individuo adulto alado (Esquivel, 2006; Raebel, Merckx, Riordan, Macdonald, \& Thompson, 2010).

La exuvia que queda en el sustrato de emergencia, constituye una fuente potencial de información indirecta de la historia natural y ecología de los odonatos (Soluk, 1990). Las exuvias se han empleado para determinar riqueza, calidad de hábitat, patrón de emergencia, proporción de sexos y densidad al momento de la emergencia (Corbet, 2004; Bried, D’Amico, \& Samways, 2012). Además, la emergencia de los insectos acuáticos constituye un evento ecológico clave en la transferencia de biomasa y energía entre ecosistemas acuáticos y terrestres (Chacón, Segnini, \& Briceño, 2016).

En Cuba, existe un número limitado de trabajos dedicados a la ecología e historia natural de los odonatos (Torres \& Fonseca, 2009; Trapero-Quintana, Reyes-Tur, \& Cuéllar, 2011, 2012). En relación a la emergencia de sus larvas, para el archipiélago cubano solo existe la referencia de Trapero-Quintana y Reyes-Tur (2010) para un hábitat léntico permanente.

Corbet $(1954,1958)$ señaló, para los odonatos del hemisferio norte, dos modelos 
de emergencia determinados por los procesos de regulación estacional que inducen o no diapausa. Un modelo incluye las especies de primavera, las cuales emergen a principio del año y tienen períodos de emergencia cortos y sincronizados. Como modelo alternativo, las especies de verano son aquellas sin diapausa con período de emergencia veraniego, largo y asincrónico (Corbet, 1954).

Para los odonatos de zonas templadas la regulación estacional de la emergencia se activa fundamentalmente por el fotoperíodo y los cambios de temperatura (Norling, 1984), donde la sincronización aumenta con la latitud. Por otro lado, en los trópicos, este evento depende en mayor cuantía de fluctuaciones en las precipitaciones (Mathavan \& Pandian, 1977), así como de la variabilidad estructural de los hábitats dulceacuícolas (Corbet, Suhling, \& Soendgerath, 2006). Estos criterios permiten inferir que la emergencia en los trópicos no es un proceso homogéneo (Suhling, 1995), sino temporalmente heterogéneo (Lutz \& McMahan, 1973). Para evaluar la veracidad de esta inferencia, el presente estudio tiene como objetivo describir la relación entre la temperatura, humedad relativa y precipitaciones con el patrón de emergencia, para especies de odonatos en un hábitat dulceacuícola lótico de Giro, en el oriente de Cuba, a partir de la recolección de exuvias. El conocimiento de la riqueza específica de odonatos y su patrón de emergencia, puede ser de utilidad para los esfuerzos conservacionistas en localidades donde son crecientes las prácticas agrícolas.

\section{MATERIALES Y MÉTODOS}

Área de estudio: Giro está ubicado al norte de la ciudad de Santiago de Cuba, en el municipio Palma Soriano (2007'42" N $76^{\circ} 02{ }^{\prime} 32^{\prime}$ W) (Fig. 1). Es un cuerpo de agua lótico permanente, con insolación filtrada por bosque secundario en ecótopo de bosque de galería (Capote \& Berazaín, 1984). Abundan individuos de Cecropia schraebaeriana Miq., Syzygium jambos (L.) Alston, Zanthoxyllum martinicense (Lam.) DC., Clusia rosea Jacq.,

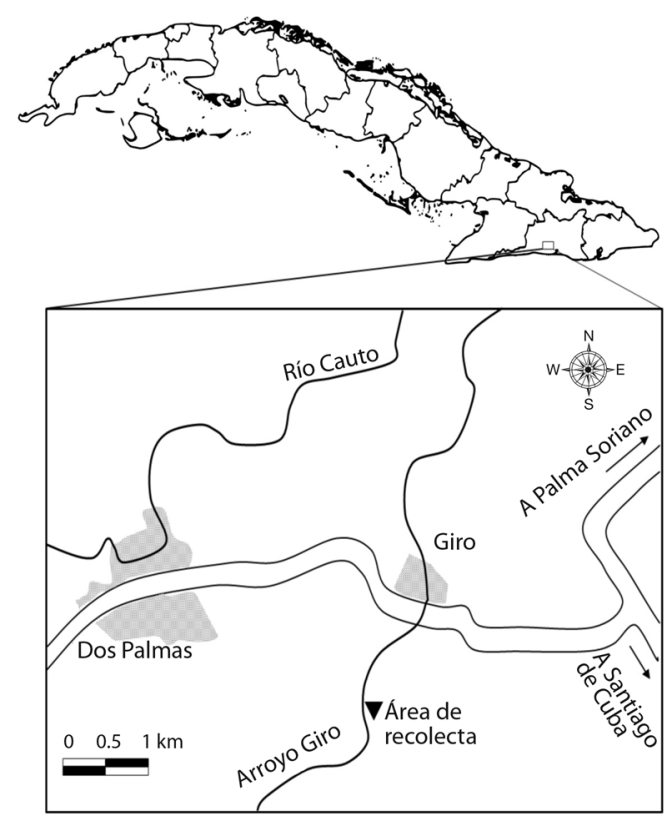

Fig. 1. Ubicación geográfica del hábitat lótico de Giro, Santiago de Cuba, Cuba.

Fig. 1. Geographical location of lotic habitat in Giro, Santiago de Cuba, Cuba.

Roystonea regia (Kunth) O. F. Cook, Melicoccus bijugatus Jacq., Guazuma ulmifolia Lam., Bursera simaruba (L.) Sargent, Albizia lebbeck (L.) Benth. y Gerascanthus gerascanthoides (Kunth) Borhidi.

La vegetación de ribera está formada esencialmente por ciperáceas, aunque existen individuos de plantas no acuáticas como Cissus verticillata (L.) Nicholson y Jarvis, Thunbergia sp., Petiveria alliacea L., Sida rhombifolia L., Passiflora sp. y Capraria biflora L. Por otra parte, Cyperus ochraceus y Caperonia palustris (L.) A. St. Hil. representan el $80 \%$ del área cubierta por las especies vegetales. El río tiene fondo arenoso gravoso, con 10 $\mathrm{m}$ de ancho promedio, $50 \mathrm{~cm}$ de profundidad máxima y $0.17 \mathrm{~m} / \mathrm{s}$ de velocidad promedio de la corriente. Además, el $50 \%$ de la superficie de ambas orillas presenta cobertura vegetal. En la época poco lluviosa se registró presencia de Cladophora sp. Adicionalmente, se detectaron los peces Gambusia punctata (Poey, 1854) y Nandopsis tetracanthus (Cuvier \& Valenciennes, 1831), entre julio y octubre de 2008. 
Las precipitaciones medias anuales históricas oscilan entre 714 - $1150 \mathrm{~mm}$ con humedad relativa promedio entre 77.8 y $86.7 \%$, con 24.8 ${ }^{\circ} \mathrm{C}$ de temperatura media anual $\left(21.4{ }^{\circ} \mathrm{C}\right.$ mínima y $26.8^{\circ} \mathrm{C}$ máxima). La velocidad promedio del viento es de $2.4 \mathrm{~km} / \mathrm{h}$. Los datos fueron proporcionados por el Centro Meteorológico Provincial de Santiago de Cuba.

Muestreo: El estudio se realizó entre enero y diciembre 2008 recolectando el material cada siete días entre 09:00 y 14:00, para evitar interferencias en la emergencia (Trottier, 1973; Corbet, 2004). La recolecta directa de las exuvias sobre la vegetación, se realizó con pinzas entomológicas, en una sección de $8 \mathrm{~m}$ de largo por $1 \mathrm{~m}$ de ancho, abarcando la transición entre el agua y la tierra (Johansson \& Brodin, 2003; Johansson, Crowley, \& Brodin, 2005). Las exuvias se preservaron en frascos etiquetados de $25 \mathrm{~mL}$ con alcohol al $70 \%$.

Identificación taxonómica: La determinación taxonómica se realizó con ayuda de claves dicotómicas (Westfall \& May, 1996; Novelo-Gutiérrez, 1997a, 1997b; Needham, Westfall, \& May, 2014; Trapero-Quintana \& Naranjo, 2009). Todo el material biológico generado por el presente estudio, se encuentra depositado en la colección entomológica del Museo de Historia Natural Charles Ramsden de la Torre de la Universidad de Oriente. La medición de la talla de las exuvias, se realizó con un microscopio estereoscópico marca Novel NTB$2 \mathrm{~B}$, con aumento de $40 \mathrm{X}$ y error de $0.5 \mathrm{~mm}$.

Análisis de la composición y estructura: La composición y abundancia se caracterizó a partir de la riqueza específica y el número de exuvias por especie, respectivamente. Para caracterizar la odonatocenosis de cada localidad, se calculó la abundancia y biomasa relativas según la ecuación:

$$
P_{i}=\frac{n_{i}}{N}
$$

donde $n_{i}$ : es el número de individuos (o la biomasa) de la especie $i$ y $N$ : es el número total de individuos (o su biomasa total). Para representar la distribución de abundancia se construyeron las gráficas de rango-abundancia o curvas de Whittaker (1965) con ejes expresados en escala logarítmica. Se consideraron especies dominantes las que presentaron más de 40 exuvias, las de abundancia intermedia entre 5 y 40 exuvias y el resto como raras. La biomasa $(B)$ se calculó a partir de la relación lineal entre el largo total (talla) y la masa, propuesta por Benke, Huryn, Smock y Wallace (1999) a través de la ecuación general:

$$
B=a+b \cdot L
$$

donde $L$ : es el largo máximo de la exuvia, $a$ : es la constante que indica el punto de intercepción con el eje Y y $b$ : el coeficiente de regresión. La ecuación particular para el orden Odonata es la que sigue:

$$
B=0.0078+2.792 \cdot L\left(r^{2}-0.95\right)
$$

Para determinar la variación temporal de la composición específica se calculó el porcentaje de constancia (PC), a través del cociente entre el número de muestreos con registros de emergencia y el total de muestreos durante el año. Las especies se clasificaron a partir de los criterios de Velasco, Millán y Ramírez (1993) en Constantes para $\mathrm{PC} \geq 50 \%$; Ocasionales, 25 $\%<\mathrm{PC}<50 \%$ y Accidentales para PC $\leq 25 \%$.

Análisis de los patrones de emergencia: De acuerdo a la caracterización climatológica de los períodos climáticos para Cuba propuesta por Lecha y Chugaev (1989), se analizó el patrón de emergencia durante los períodos lluvioso, desde marzo hasta octubre; poco lluvioso, desde diciembre a marzo; y de transición, abril y noviembre.

Se elaboraron histogramas de emergencia por especie a partir del número de exuvias por mes. Se confeccionó una matriz de trabajo que incluyó como variables el número de exuvias, la frecuencia de emergencia en cada época del año y el número de picos con valor mayor o igual a $50 \%$ de emergencia por períodos 
climáticos. Se definió como período de emergencia notable, cuando los valores de emergencia fueron mayores o iguales a $50 \%$ del total, y emergencia máxima al mayor registro de exuvias para cada especie, durante el año de muestreo (Mathavan \& Pandian, 1977).

La caracterización de las variables se realizó a través del cálculo de media y desviación estándar. La normalidad de las variables se verificó mediante la prueba de bondad de ajuste $\mathrm{D}_{\text {máxima }}$ de Kolmogorov-Smirnov. Para detectar posibles ensambles de especies en relación con la emergencia, se realizó el análisis de similitud utilizando el coeficiente de Bray-Curtis, a partir de la frecuencia de exuvias emergidas por temporada del año y meses de transición. Para determinar el grado de asociación entre las variables climáticas con las especies de Odonata y entre el número de individuos de cada especie, se realizó un análisis de componentes principales. La elección de los componentes se efectuó mediante el criterio de Kaiser-Guttman, con el análisis de los componentes con valor propio igual o mayor que 1 (Jackson, 1993). Los cálculos estadísticos se realizaron mediante el programa STATISTICA ${ }^{\circledR}$ 8.0, StatSoft, excepto la similitud para la cual se utilizó el programa PRIMER 6.0.

\section{RESULTADOS}

Composición y curvas de rango-abundancia: En la localidad de estudio se registraron 22 especies $(26 \%$ del total de registros

\section{CUADRO 1}

Porcentaje de exuvias $(\mathrm{N}=443)$ y de biomasa de las especies de Odonata recolectadas entre enero y diciembre 2008, en un hábitat lótico en Giro, Santiago de Cuba, Cuba

TABLE 1

Percentage of exuvias $(\mathrm{N}=443)$ and biomass of Odonata species collected between January and December 2008, in a lotic habitat at Giro, Santiago de Cuba, Cuba

\begin{tabular}{|c|c|c|c|c|}
\hline Suborden & Familias & Especies & Porcentaje de exuvias & Biomasa \\
\hline \multirow{7}{*}{ 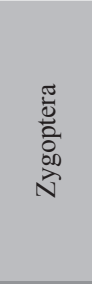 } & \multirow[t]{2}{*}{ Lestidae } & Lestes forficula Rambur, 1842 & 0.2 & 0.002 \\
\hline & & Lestes tenuatus (Rambur, 1842) & 1.7 & 0.02 \\
\hline & \multirow[t]{2}{*}{ Protoneuridae } & Neoneura maria (Scudder, 1866)* & 9.9 & 0.1 \\
\hline & & Protoneura capillaris (Rambur 1842)* & 17 & 0.2 \\
\hline & \multirow[t]{3}{*}{ Coenagrionidae } & Enallagma coecum (Hagen, 1861) & 6.3 & 0.1 \\
\hline & & Ischnura hastata (Say, 1839) & 0.5 & 0.004 \\
\hline & & Telebasis dominicanum (Selys, 1857)** & 0.2 & 0.001 \\
\hline \multirow{15}{*}{ 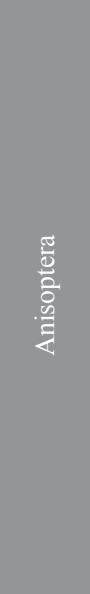 } & \multirow[t]{3}{*}{ Aeshnidae } & Gynacantha nervosa Rambur, 1842 & 0.7 & 0.01 \\
\hline & & Rhionaeschna psilus (Calvert, 1947) & 1.6 & 0.03 \\
\hline & & Triacanthagyna septima (Selys, 1857) & 0.2 & 0.002 \\
\hline & Gomphidae & Progomphus integer Hagen in Selys, 1878** & 5.8 & 0.1 \\
\hline & \multirow[t]{11}{*}{ Libellulidae } & Crocothemis servilia (Drury, 1770) & 0.7 & 0.01 \\
\hline & & Dythemis rufinervis (Burmeister, 1839)** & 5.6 & 0.1 \\
\hline & & Erythrodiplax berenice naeva (Hagen, 1861) & 0.2 & 0.002 \\
\hline & & Erythrodiplax justiniana (Selys, 1857)** & 0.2 & 0.002 \\
\hline & & Erythrodiplax umbrata (Linnaeus, 1758) & 0.5 & 0.01 \\
\hline & & Macrothemis celeno (Selys, 1857)** & 37 & 0.4 \\
\hline & & Orthemis ferruginea (Fabricius, 1775) & 1.8 & 0.02 \\
\hline & & Pantala flavescens (Fabricius, 1798) & 2.7 & 0.04 \\
\hline & & Pantala hymenaea (Say, 1839) & 0.2 & 0.002 \\
\hline & & Perithemis domitia (Drury,1773) & 2.9 & 0.03 \\
\hline & & Scapanea frontalis (Burmeister, 1839)** & 4.1 & 0.04 \\
\hline
\end{tabular}

\footnotetext{
* Especies endémicas de Cuba (Cuban endemic species), ** Especies endémicas de las Antillas (Antillean endemic species).
} 
para Cuba); siete de Zygoptera (36\%) y 15 de Anisoptera (64\%), pertenecientes a seis familias y 18 géneros. Se recolectaron 443 exuvias (68\% Anisoptera y $32 \%$ de Zygoptera), promediado en $37 \pm 2$ exuvias por mes. La familia mejor representada fue Libellulidae con 11 especies $(50 \%)$ y 247 exuvias $(56 \%)$ (Cuadro 1). Erythrodiplax resultó el género con mayor aporte específico con tres especies. El endemismo en esta localidad estuvo representado por Protoneura capillaris y Neoneura maria, lo que significó el $40 \%$ de los cinco endémicos para Cuba.

La curva de rango-abundancia de especies, representa una odonatocenosis de distribución poco equitativa (Fig. 2A). Macrothemis celeno está situada en el extremo superior con 164 exuvias, seguida por $P$. capillaris con 77 y $N$. maria con 44, que sumadas significan el $64 \%$ del total. A continuación se ubicaron las especies de abundancia intermedia con registros entre siete y 28 exuvias, cuya contribución se encuentra entre 1.7 y $6.3 \%$ (Cuadro 1; Fig. 2A). En la última porción de la curva se ubicaron especies con registros entre una y tres exuvias, clasificadas como raras (Fig. 2A). Por otro lado, la abundancia en función de la biomasa también responde a una distribución no equitativa, para las tres especies dominantes de abundancia relativa (Fig. 2B). Estas últimas con aportes entre $10 \%$ y $40 \%$, representan el $70 \%$ de la biomasa total.

La odonatofauna de este hábitat está constituida básicamente por especies Accidentales (86\%). M. celeno resultó Constante con 50 $\%$, mientras $P$. capillaris y E. coecum, con 38 $\%$ y $33 \%$ respectivamente, clasificaron como Ocasionales (Cuadro 2).

Patrones de emergencia: En relación al número de exuvias por mes, ocho especies presentaron valores máximos entre cinco y 20 exuvias por muestreo (Fig. 3A, Fig. 3B, Fig. 3C, Fig. 3D, Fig. 3E, Fig. 3F, Fig. 3G, Fig. $3 \mathrm{H})$. Se detectó segregación temporal de picos de emergencia para $P$. capillaris, N. maria, $P$. integer, M. celeno y $S$. frontalis. Para las especies con registro entre una y cuatro exuvias
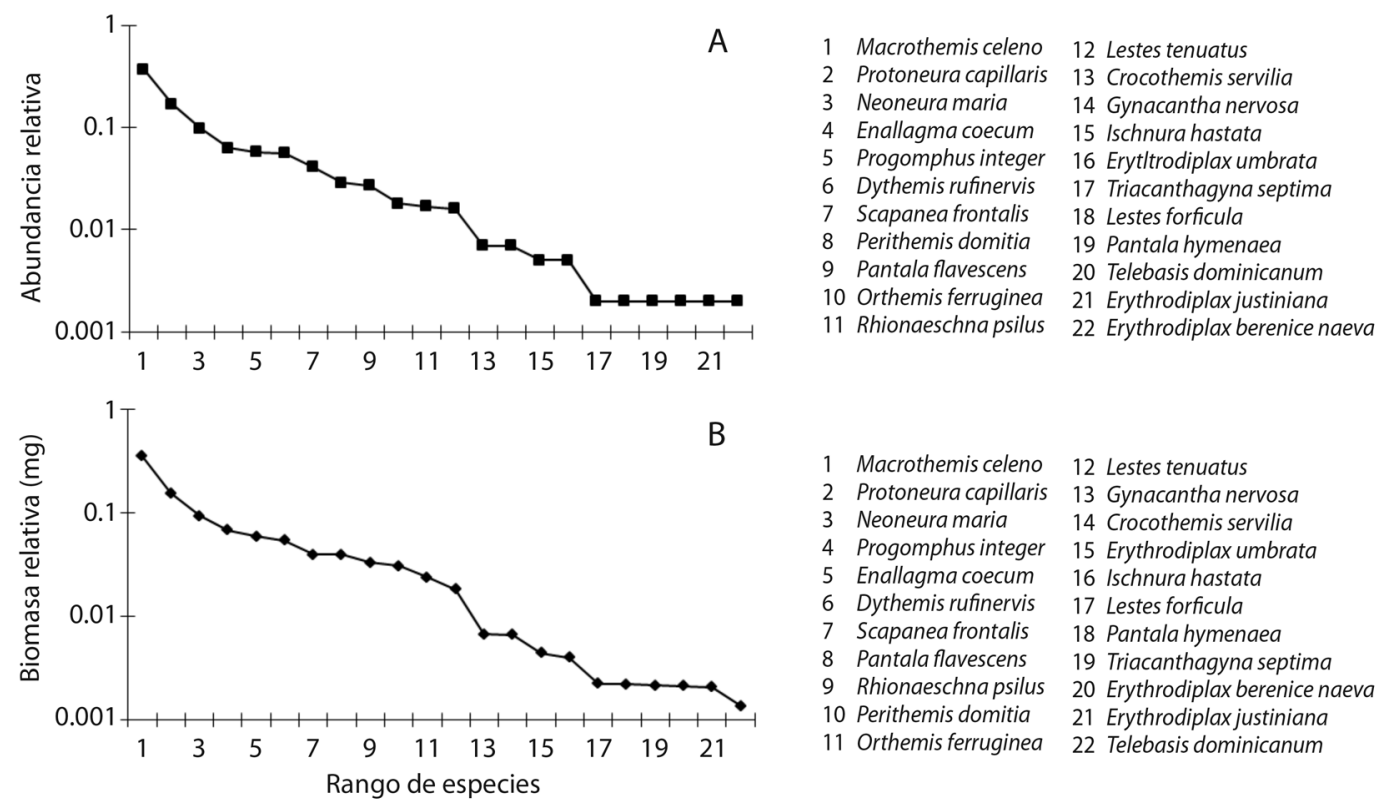

\footnotetext{
1 Macrothemis celeno 12 Lestes tenuatus 2 Protoneura capillaris 13 Gynacantha nervosa 3 Neoneuramaria 14 Crocothemis servilia 4 Progomphusinteger 15 Erythrodiplax umbrata 5 Enallagmacoecum 16 Ischnura hastata 6 Dythemis rufinervis 17 Lestesforficula 7 Scapaneafrontalis 18 Pantala hymenaea 8 Pantala flavescens 19 Triacanthagyna septima 9 Rhionaeschnapsilus 20 Erythrodiplax berenice naeva 10 Perithemis domitia 21 Erythrodiplax justiniana 11 Orthemis ferruginea 22 Telebasis dominicanum
}

Fig. 2. Curva de rango-abundancia (A) y biomasa relativa (B), para las especies de Odonata recolectadas entre enero y diciembre 2008, en un hábitat lótico de Giro, Santiago de Cuba, Cuba.

Fig. 2. Curves of rank abundance (A) and relative biomass (B) of Odonata species collected between January and December 2008, in a lotic habitat at Giro, Santiago de Cuba, Cuba. 


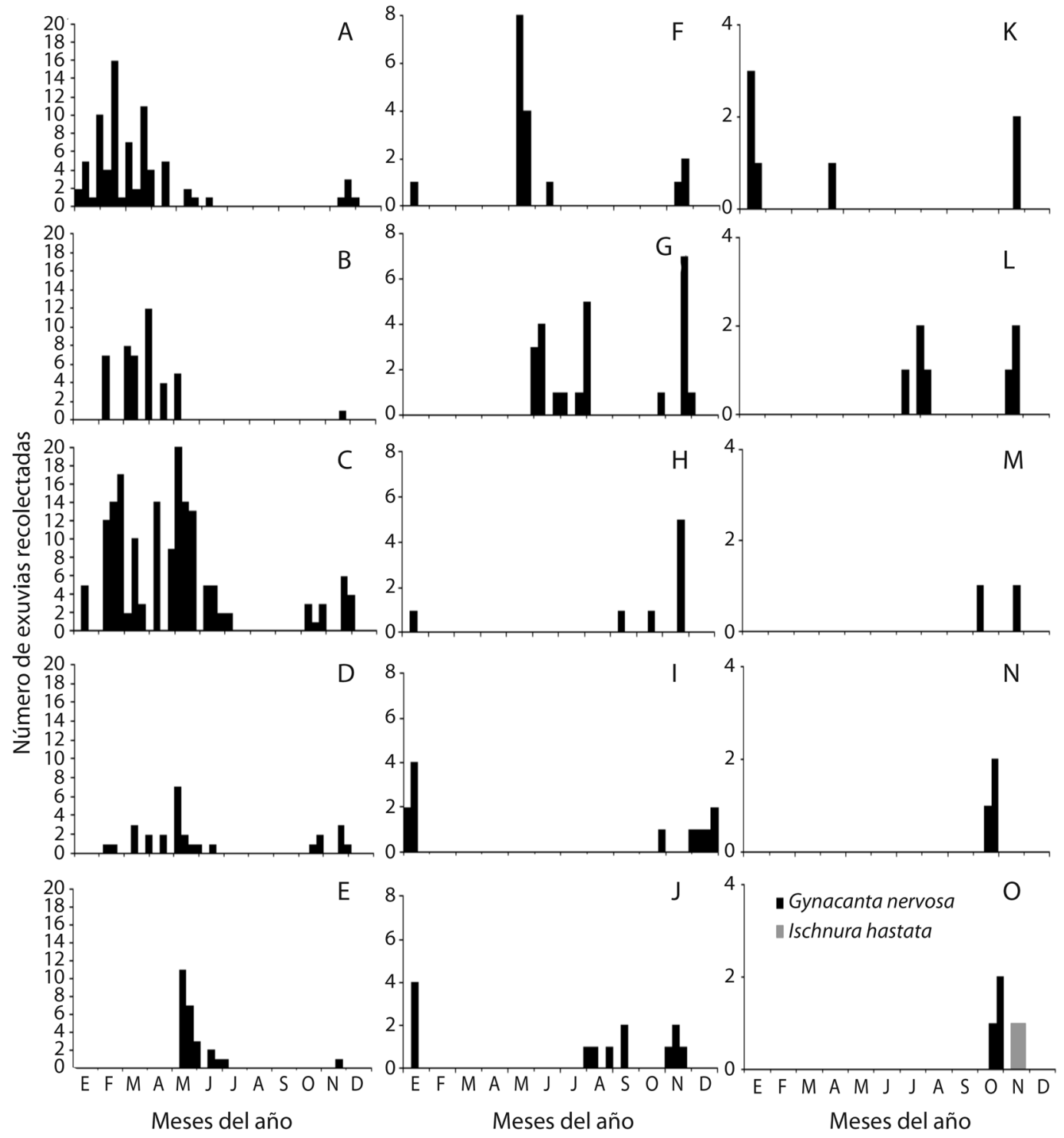

Fig. 3. Histogramas de emergencia para las especies de Odonata recolectadas entre enero y diciembre 2008, en un hábitat lótico de Giro, Santiago de Cuba, Cuba. (Se excluyeron las especies que presentaron una exuvia).

Fig. 3. Histogram of emergence of Odonata species collected between January and December 2008, in a lotic habitat at Giro, Santiago de Cuba, Cuba. A: Protoneura capillaris, B: Neoneura maria, C: Macrothemis celeno, D: Enallagma coecum, E: Progomphus integer, F: Scapanea frontalis, G: Dythemis rufinervis, H: Orthemis ferruginea, I: Pantala flavescens, J: Perithemis domitia, K: Rhionaeschna psilus, L: Lestes tenuatus, M: Erythrodiplax umbrata, N: Crocothemis servilia, O: Gynacantha nervosa e Ischnura hastate (Species with only one exuviae were omitted). 
CUADRO 2

Presencia de exuvias y porcentaje de constancia (PC) para las especies de Odonata recolectadas entre enero y diciembre 2008, en un hábitat lótico de Giro, Santiago de Cuba, Cuba

TABLE 2

Exuviae presence and constancy percentage (PC) of Odonata species collected between January and December 2008, in a lotic habitat at Giro, Santiago de Cuba, Cuba

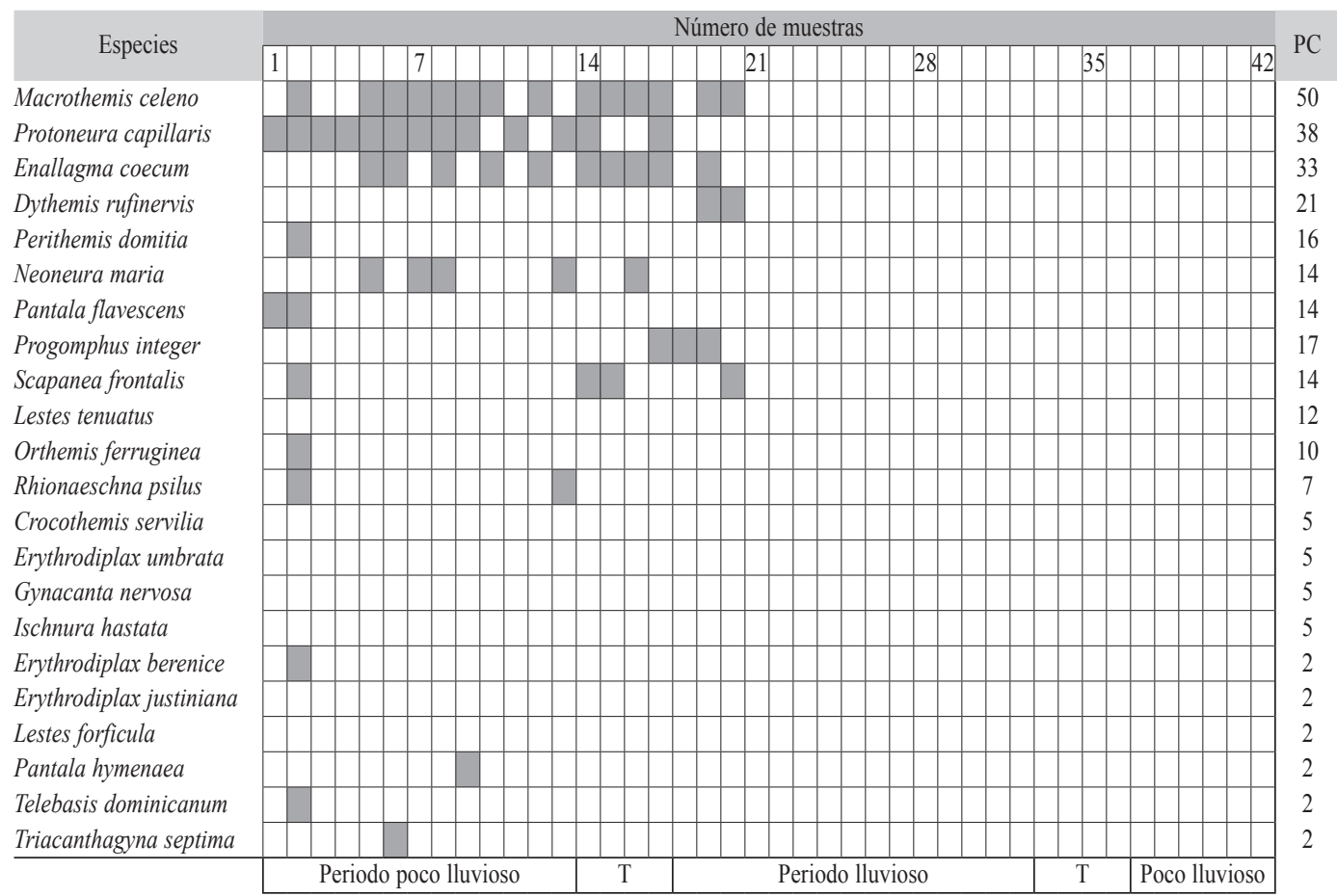

T: muestreo durante los meses de transición abril y noviembre (T: sample during transition months, April and November). (Clasificación del porcentaje de constancia: constante PC $\geq 50 \%$, ocasional, $25 \%<\mathrm{PC}<50 \%$ y accidental, $\mathrm{PC} \leq 25 \%$; gris: muestreos con presencia de exuvias, blanco: muestreo sin exuvias).

(Constancy percentage classification: constant $\mathrm{PC} \geq 50 \%$, occasional, $25 \%<\mathrm{PC}<50 \%$ y accidental, $\mathrm{PC} \leq 25 \%$; gray: samples with exuviae presence, White: samples without exuviae).

por muestreo, predominó la emergencia temporalmente dispersa (Fig. 3I, Fig. 3J, Fig. 3K, Fig. 3L, Fig. 3M, Fig. 3N, Fig. 3O). M. celeno y E. coecum, sincronizaron los picos de emergencia en mayo con 20 y siete individuos respectivamente (Fig. 3D y Fig. 3G). En enero P. flavescens y $P$. domitia presentaron sincronía de emergencia máxima con igual número de exuvias (cuatro registros) (Fig. 3I y Fig. 3J).

Los picos máximos de emergencia se observaron en el periodo poco lluvioso, donde se registró el $50 \%$ del total de exuvias respecto a la temporada lluviosa para un $18 \%$.
En febrero se detectó la mayor cantidad de emergencias con 112 exuvias (25\%), seguido por abril con 95 (22\%) (Fig. 3). En septiembre se registró $O$. ferruginea y $P$. domitia con una y dos exuvias respectivamente (Fig. 3).

En el análisis de componentes principales, los dos primeros ejes representan el $69.9 \%$ de la varianza total (Cuadro 3). En el primer componente, con aporte de $42.3 \%$, los mayores factores de carga indican la relación inversamente proporcional, entre la variación del número de exuvias y las variables climáticas, excepto con la temperatura máxima (Cuadro 3). 
CUADRO 3

Análisis de Componentes Principales para la relación entre el número de exuvias y especies de Odonata recolectadas, con las variables climáticas entre enero y diciembre 2008, en un hábitat lótico de Giro, Santiago de Cuba, Cuba

TABLE 3

Principal component analysis of exuviae number and Odonata species collected between January and December 2008, in a lotic habitat at Giro, Santiago de Cuba, Cuba

\begin{tabular}{lccc}
\multicolumn{1}{c}{ Variables } & \multicolumn{3}{c}{ Componentes } \\
Número de exuvias & $\mathbf{0 . 6 5}$ & 0.44 & -0.35 \\
Número de especies & 0.31 & $\mathbf{0 . 5 7}$ & -0.70 \\
Temperatura promedio & $\mathbf{- 0 . 6 3}$ & $\mathbf{- 0 . 6 7}$ & -0.37 \\
Temperatura máxima & -0.22 & $\mathbf{- 0 . 9 0}$ & -0.24 \\
Temperatura mínima & $\mathbf{- 0 . 8 5}$ & -0.31 & -0.38 \\
Humedad relativa promedio & $\mathbf{- 0 . 7 7}$ & $\mathbf{0 . 5 4}$ & -0.06 \\
Humedad relativa máxima & $\mathbf{- 0 . 7 7}$ & 0.09 & 0.13 \\
Humedad relativa mínima & $\mathbf{- 0 . 6 8}$ & $\mathbf{0 . 5 9}$ & -0.16 \\
Precipitaciones & $\mathbf{- 0 . 6 7}$ & 0.26 & 0.25 \\
Días lluviosos & $\mathbf{- 0 . 6 6}$ & 0.43 & 0.05 \\
Valores propios & 4.2 & 2.8 & 1.1 \\
Varianza (\%) & 42.3 & 27.6 & 10.5 \\
Varianza acumulada (\%) & 42.3 & 69.9 & 80.5 \\
\hline
\end{tabular}

${ }^{1}$ La elección de los componentes se efectuó mediante el criterio de Kaiser-Guttman (Jackson, 1993).

${ }^{1}$ The choice of the components was done by the KaiserGuttman criteria (Jackson, 1993).

Por otro lado, el segundo componente explica la interacción entre el número de especies con la temperatura promedio y máxima, así como con la humedad relativa promedio y mínima, con un aporte de $27.6 \%$. El tercer componente no tiene aporte significativo en los resultados.

A partir del análisis de agrupamiento se diferenciaron tres grupos (Fig. 4). El primero incluyó 14 especies (entre P. capillaris y E. umbrata), que emergieron en al menos uno de los meses de transición. Es importante resaltar que el subgrupo que abarcó desde $D$. rufinervis hasta $E$. umbrata está constituido por las especies con emergencia máxima en el periodo lluvioso. Las especies de los restantes dos grupos nunca emergieron en abril o noviembre y siempre se detectaron en una de las temporadas (Fig. 4).

\section{DISCUSIÓN}

La mayor riqueza específica observada para el suborden Anisoptera, corresponde con un patrón común en las zonas tropicales (Corbet, 2004; Alonso-EguíaLis, 2004), tanto en hábitats lénticos como lóticos. Una explicación posible está en la capacidad de muchos anisópteros, en particular especies de Libellulidae, en desarrollar sus larvas tanto en ríos, arroyos de montañas y lagunas permanentes o temporales. Además, a nivel mundial esta es la familia con mayor número de especies (Garrison, von Ellenrieder, \& Louton, 2006). Por otra parte, Erythrodiplax es considerado uno de los géneros más diversos de América, con más de la mitad de sus especies como propias del continente (Esquivel, 2006). De los odonatos endémicos del archipiélago, las dos especies observadas corresponden con los registros para Cuba oriental en esta categoría (Trapero-Quintana \& Naranjo, 2003).

De las especies registradas, $M$. celeno, $P$. capillaris, N. maria, P. integer y D. rufinervis, son abundantes en ríos con bosques de galería, lo cual se comprobó por su dominancia en función de la abundancia y la biomasa. Estos resultados se corresponden con los registros de Puerta (2002), Trapero-Quintana y Naranjo (2003), Hernández y Cala (2003) y Rodríguez y González (2004) para ríos del centro y del oriente de Cuba; así como Novelo-Gutiérrez (2007) y Garrison, von Ellenrieder y Louton (2010) para América Central y del Sur.

Tanto M. celeno (Constante) y P. capillaris (Ocasional), han sido observadas con mayor frecuencia en hábitats lóticos (Alayo, 1968; Westfall \& May, 1996; Needham et al., 2014). Por otro lado, el hecho que N. maria, P. integer y $S$. frontalis resultaran Accidentales, puede ser causado por su frecuente asociación con los ambientes lóticos al tener valores de profundidad superiores a $50 \mathrm{~cm}$ y de mayor cobertura por hidrófitas (Alayo, 1968; Daigle, 1993; Trapero-Quintana \& Naranjo, 2003). Todo esto sugiere tener precaución al clasificar las especies estrictamente para un determinado hábitat. 


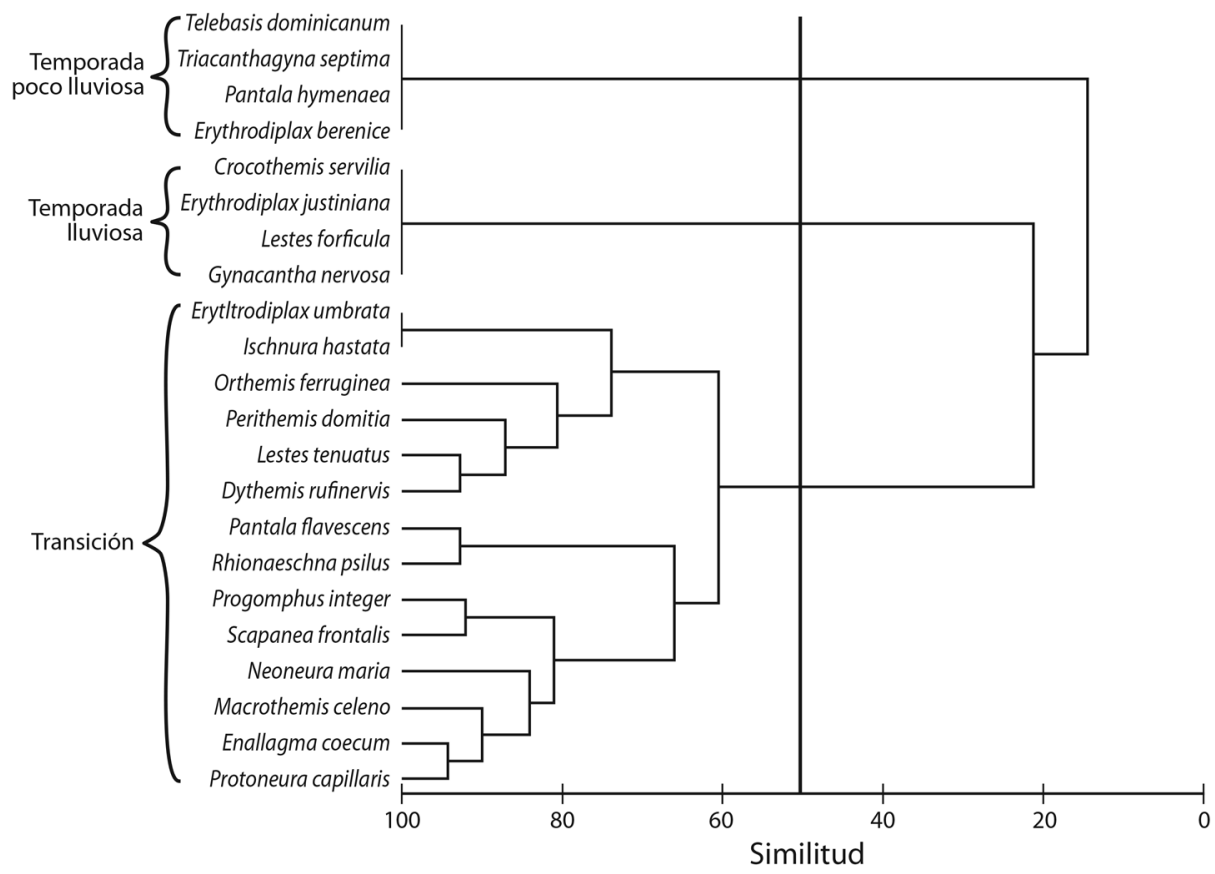

Fig. 4. Análisis de similitud basado en el coeficiente de Bray-Curtis entre las especies de Odonata recolectadas entre enero y diciembre 2008, en un hábitat lótico de Giro, Santiago de Cuba, Cuba. (La línea perpendicular al eje horizontal representa el $50 \%$ del coeficiente de similitud, que constituye el criterio general para delimitar los grupos señalados).

Fig. 4. Bray-Curtis coefficient-based similarity analysis of Odonata species collected between January and December 2008, in a lotic habitat at Giro, Santiago de Cuba, Cuba. (The vertical line on the horizontal axis represents a similarity coefficient of $50 \%$, the criterion for discriminating the highlighted groups).

Partiendo de los modelos de emergencia propuestos por Corbet (1954, 1958), según las condiciones ambientales de Cuba, los resultados apuntan hacia patrones típicos de especies que al parecer no experimentan diapausa en el último estadio larvario. Además, no presentan un patrón homogéneo de emergencia, como afirma Suhling (1995) para los trópicos. Al mismo tiempo, la sincronía solo fue detectada para los picos máximos de emergencia, entre las especies que pertenecen a diferentes grupos morfofuncionales. Estas evidencias apoyan la veracidad de la inferencia inicial sobre la heterogeneidad temporal de la emergencia de odonatos en los trópicos (Lutz \& McMahan, 1973). Sin embargo, en las condiciones de ecosistemas insulares, parece insostenible la clasificación estricta de modelos de emergencia para especies de primavera y verano tal como fue propuesta por Corbet $(1954,1958)$.
La mayoría de las especies emergieron durante los meses de transición o en ambos períodos climáticos, similar a los resultados obtenidos para odonatos en un hábitat léntico permanente de Cuba oriental (Trapero-Quintana \& Reyes-Tur, 2010).

Por otro lado, para efemerópteros de los Andes venezolanos, también se registraron patrones de emergencia no homogéneos, con segregación temporal de los picos máximos (Chacón et al., 2016), pese a las diferencias de temperaturas con el hábitat objeto de estudio. Asimismo, para Anopheles aquasalis (Diptera) en Venezuela se registró que la abundancia de sus larvas está directamente relacionada con el período lluvioso, sin embargo, la misma puede variar de acuerdo al tipo de hábitat (Berti et al., 2010). Por otro lado, TorresGarcía, Pérez-Valladares, Herrería-Diego y Pineda-López (2014), obtuvieron resultados 
que indican diferenciación en el período de emergencia para insectos hemimetábolos y coleópteros acuáticos, en la cuenca del río Xichú, Guanajuato, México.

El mayor porcentaje de emergencia para la temporada poco lluviosa, pudiera ser un reflejo de la dinámica del hábitat lótico estudiado, respecto a los niveles y el flujo del caudal, así como a la profundidad del río. La crecida de los ríos debido a las lluvias, provoca el arrastre de las larvas y las plantas acuáticas, con la modificación significativa de los microhábitats $\mathrm{y}$ sustratos de emergencia. Esto se relaciona con el mayor número de emergencias detectadas durante el periodo poco lluvioso, debido a la mayor estabilidad del volumen hídrico del río. En ensambles de odonatos en hábitat lóticos de la cuenca hidrográfica de Aragón España, las lluvias fueron consideradas como la variable determinante en la baja abundancia de odonatos, por las crecidas de los ríos (Torralba-Burrial, 2008).

La emergencia se caracterizó por la sincronía de picos notables para especies de diferentes grupos morfofuncionales, según la clasificación de Trapero-Quintana et al. (2012). Por ejemplo, $M$. celeno que pertenece al grupo Agarradores, mostró sincronía con E. coecum, cuyas larvas son clasificadas como Hidrofitobiontes, ya que se desarrollan generalmente entre la vegetación acuática. Esto último se corresponde con la poca equitatividad en la distribución de abundancia específica y el elevado porcentaje de especies Accidentales detectadas.

Por otro lado, la emergencia significativa de los odonatos en la época poco lluviosa, podría deberse a la disminución de la precipitación, con la consecuente reducción de la disponibilidad de alimentos y con ello la disminución de las especies competidoras o depredadoras. Sin embargo, no se descarta que la precipitación de los días previos a los muestreos tenga un efecto sinérgico, ya que durante la emergencia, el porcentaje elevado de la humedad relativa es indispensable, lo cual contribuye a impedir la deshidratación de los tenerales. A favor de este planteamiento el primer autor ha registrado valores de humedad relativa entre 71 y $80 \%$ durante la emergencia de N. maria (A. Trapero, datos no publicados).

La relación entre la frecuencia de exuvias y de especies con la temperatura y la humedad relativa, también se demostró mediante el análisis de componentes principales. La temperatura mantuvo el mayor valor absoluto de factor de carga en el componente que mejor explica la variación del número de exuvias. En correspondencia con estos resultados Chacón et al. (2016) para el orden Ephemeroptera, concluyeron que los periodos de emergencia en estos insectos, eran el resultado de ambientes térmicamente favorables para su óptimo desarrollo.

El análisis de agrupamiento resultó de gran importancia al determinar la tendencia general del patrón temporal de emergencia. El dendrograma confeccionado a partir de la frecuencia de las exuvias emergidas por temporada climática, arrojó un grupo de especies exclusivas del periodo lluvioso y otro cuya emergencia abarcó al menos uno de los meses de transición. Esta tendencia demuestra la heterogeneidad temporal de la emergencia para las especies estudiadas, descarta la posibilidad de sincronía del inicio o culminación de la emergencia y sin diapausa para las especies. Aunque el patrón de emergencia está relacionado con la variación de la temperatura, la humedad relativa y las precipitaciones, consideramos que su importancia relativa depende de su interacción con las características del hábitat y su dinámica.

\section{AGRADECIMIENTOS}

Los autores reconocen la colaboración brindada por los colegas Alberto Cabrera Anaya, Roger Rivero González y José Alberto Pérez Remesal que participaron en la recolecta de exuvias. Además, agradecemos la colaboración de Nilia Cuéllar Araújo y John A. Allen en la lectura crítica del manuscrito y a Yiezenia Rosario Ferrer por su ayuda en la calidad de las figuras. Especial reconocimiento merecen los revisores y editores cuyas críticas y sugerencias ayudaron a mejorar notablemente la primera versión del manuscrito. 


\section{RESUMEN}

Se conoce poco sobre los patrones de emergencia de los odonatos tropicales. Estudiamos el patrón de emergencia de odonatos en un cuerpo dulceacuícola lótico de Giro, al norte de la ciudad de Santiago de Cuba, entre enero y diciembre 2008. Visitamos la localidad semanalmente, entre 09:00 y 14:00 h y recolectamos las exuvias directamente sobre la vegetación de ribera en una sección fija de $8 \times 1 \mathrm{~m}^{2}$. Caracterizamos la variación temporal de la composición específica, la abundancia y la biomasa relativas y el patrón de emergencia de cada especie. Registramos 443 exuvias, pertenecientes a 22 especies, siete de Zygoptera y 15 de Anisoptera. El $50 \%$ de la emergencia anual ocurrió en la temporada poco lluviosa (diciembre a marzo) con el valor más elevado en febrero (25\%). En el caso de las especies con siete o más exuvias por mes, Enallagma coecum y Macrothemis celeno se sincronizaron temporalmente durante la emergencia. También encontramos segregación temporal entre Macrothemis celeno con Protoneura capillaris, Neoneura maria, Progomphus integer y Scapanea frontalis. Estas diferencias se relacionaron con las mayores fluctuaciones anuales de temperatura, humedad relativa y el número de días lluviosos por mes. En general, detectamos asincronía y heterogeneidad en los patrones temporales de emergencia de los odonatos del cuerpo dulceacuícola lótico estudiado.

Palabras clave: Odonata, exuvia, patrones de emergencia, factores climáticos, Cuba.

\section{REFERENCIAS}

Alayo, P. (1968). Las libélulas de Cuba. (Insecta: Odonata). Torreia, 2, 3-102.

Alonso-EguíaLis, P. (2004). Ecología de las asociaciones de Odonata en el área de influencia de las microcuencas afectadas por la presa Zimapán, Querétaro e Hidalgo, México (Tesis Doctoral). Universidad Autónoma de Querétaro, Querétaro, México.

Benke, A., Huryn, A., Smock, L., \& Wallace, J. (1999). Length-mass relationships for freshwater macroinvertebrates in North America with particular reference to the southeastern United States. Journal of the North American Benthological Society, 18, 308-343.

Berti, J., González, J., Navarro-Bueno, E., Zoppi, E., Gordon, E., \& Delgado, L. (2010). Estacionalidad de la densidad larval del mosquito Anopheles aquasalis (Diptera: Culicidae) y otros insectos asociados a su hábitat en Sucre, Venezuela. Revista de Biología Tropical, 58(2), 777-787.

Bried, J. T., D’Amico, F., \& Samways, M. (2012). A critique of the dragonfly delusion hypothesis: why sampling exuviae does not avoid bias. Insect Conservation and Diversity, 5, 398-402.
Capote, R., \& Berazaín, R. (1984). Clasificación de las formaciones vegetales de Cuba. Revista del Jardín Botánico Nacional, 5, 1-49.

Chacón, M. M., Segnini, S., \& Briceño, D. (2016). Temperatura y emergencia diaria de siete géneros de Ephemeroptera (Insecta) en un río de la Selva Nublada de los Andes Tropicales. Revista de Biología Tropical, 64, 133-146.

Corbet, P. S. (1954). Seasonal regulation in British dragonflies. Nature, 174, 25-39.

Corbet, P. S. (1958). Temperature in relation to seasonal development of British dragonflies (Odonata). The Canadian Entomologist, 2, 755-757.

Corbet, P. S. (2004). Dragonflies, behavior and ecology of Odonata. Revised edition. Essex, United Kingdom: Harley Books.

Corbet, P. S., Suhling, F., \& Soendgerath, D. (2006). Voltinism of Odonata: a review. International Journal of Odonatology, 9, 1-44.

Daigle, J. J. (1993). A checklist of the Odonata of the Dominican Republic by province. Argia, 1, 65-69.

Esquivel, C. (2006). Libélulas de Mesoamérica y el Caribe. San José, Costa Rica: INBio.

Garrison, R. W., von Ellenrieder, N., \& Louton, J. A. (2006). Dragonfly genera (Odonata: Anisoptera) of the New World. An illustrated and annotated key to the Anisoptera. Baltimore: The John Hopkins University Press.

Garrison, R. W., von Ellenrieder, N., \& Louton, J. A. (2010). Damselfly genera of the New World. An illustrated and annotated key to the Zygoptera. Baltimore: The John Hopkins University Press.

Hernández, D. \& Cala, Y. (2003). Macrofauna dulceacuícola de invertebrados y calidad de las aguas del río Sevilla, Niquero, Granma (Tesis de Licenciatura). Universidad de Oriente, Santiago de Cuba, Cuba.

Jackson, D. (1993). Stopping rules in principal components analysis: a comparison of heuristical and statistical approaches. Ecology, 74, 2204-2214.

Johansson, F., \& Brodin, T. (2003). Effects of fish predators and abiotic factors on dragonfly community structure. Journal Freshwater Ecology, 18, 415-423.

Johansson, F., Crowley, P. H., \& Brodin, T. (2005). Sexual size dimorphism and sex ratios in dragonflies. Biological Journal of the Linnean Society, 86, 507-513.

Lecha, L. \& Chugaev, A. (1989). La bioclimatología y algunas de sus aplicaciones en condiciones de clima tropical húmedo. La Habana: Academia. 
Lutz, P., \& McMahan, E. (1973). Five year patterns of emergence in Tetragoneuria cynosura and Gomphus exilis (Odonata). Annals of the Entomological Society of America, 66, 1343-1348.

Mathavan, S., \& Pandian, T. J. (1977). Patterns of emergence, import of egg energy and energy export via emerging dragonfly populations in a tropical pond. Hydrobiologia, 54, 257-272.

Needham, J., Westfall, M., \& May, M. (2014). Dragonflies of North America. Gainesville, Florida: Scientific Publishers.

Norling, U. (1984). The life cycle and larval photoperiodic response of Coenagrion hastulatum (Charpentier) in two climatically different areas (Zygoptera: Coenagrionidae). Odonatologica, 13, 429-449.

Novelo-Gutiérrez, R. (1997a). Clave para la separación de familias y géneros de las náyades de Odonata de México. Parte I. Zygoptera. Dugesiana, 4, 1-10.

Novelo-Gutiérrez, R. (1997b). Clave para la determinación de familias y géneros de las náyades de Odonata de México. Parte II. Anisoptera. Dugesiana, 4, 31-40.

Novelo-Gutiérrez, R. (2007). Progomphus lambertoi (Anisoptera: Gomphidae), a new species from Mexico. Proceedings of the Entomological Society of Washington, 109, 791-797.

Puerta, Y. (2002). Caracterización de la macrofauna dulceacuícola del río Táyaba, Trinidad, Sancti Spíritus (Tesis de Licenciatura). Universidad de Oriente, Santiago de Cuba, Cuba.

Raebel, E. M., Merckx, T., Riordan, P., Macdonald, D. W., \& Thompson, D. J. (2010). The dragonfly delusion: why it is essential to sample exuviae to avoid biased surveys. Journal of Insect Conservation, 14, 523-533.

Rodríguez, Y., \& González, K. (2004). Caracterización de la macrofauna dulceacuícola del río Guaso, municipio Guantánamo (Tesis de Licenciatura). Universidad de Oriente, Santiago de Cuba, Cuba.

Soluk, D. (1990). Postmolt susceptibility of Ephemerella larvae to predatory stoneflies - constraints on defensive armor. Oikos, 58, 336-342.

Suhling, F. (1995). Temporal patterns of emergence of the riverine dragonfly Onychogomphus uncatus (Odonata: Gomphidae). Hydrobiologia, 302, 113-118.

Torralba-Burrial, A. (2008). Estado ecológico, comunidades de macroinvertebrados y de odonatos de la red fluvial de Aragón (Tesis Doctoral). Universidad de Oviedo, Oviedo, España.

Torres, Y., \& Fonseca, R. (2009). Reproductive behavior of the damselfly Hypolestes trinitatis (Gundlach) (Odonata: Megapodagrionidae) in Cuba. Odonatologica, $38,247-253$.

Torres-García, U., Pérez-Valladares, C., Herrería-Diego, Y., \& Pineda-López, Y. (2014). Efecto de los factores ambientales sobre la diversidad de insectos hemimetábolos y coleópteros acuáticos en la cuenca del Río Xichú, Guanajuato, México. Revista de Biología Tropical, 62(2), 69-80.

Trapero-Quintana, A., \& Naranjo, C. (2003). Revision of the order Odonata in Cuba. Bulletin of American Odonatology, 2, 23-40.

Trapero-Quintana, A., \& Naranjo, C. (2009). Clave para la identificación de especies de Odonata en estado larval de Cuba. Boletín Sociedad Entomológica Aragonesa, 44, 459-467.

Trapero-Quintana, A., \& Reyes-Tur, B. (2010). Patrones de emergencia de Odonata en Las Cuabas, Santiago de Cuba. Boletín Sociedad Entomológica Aragonesa, 46, 273-280.

Trapero-Quintana, A., Reyes-Tur, B., \& Cuellar, N. (2011). Esfuerzo de muestreo necesario para estimar la riqueza específica máxima en tres comunidades de Odonata en Cuba empleando exuvias. Boletín Sociedad Entomológica Aragonesa, 49, 285-290.

Trapero-Quintana, A., Reyes-Tur, B., \& Cuellar, N. (2012). Morphofunctional groups of Odonata larvae in three freshwater ecosystems from eastern Cuba. Odonatologica, 41, 135-150.

Trottier, R. (1973). Influence of temperature and humidity on the emergence behaviour of Anax junius. Canadian Entomology, 105, 975-984.

Velasco, J., Millán, A., \& Ramírez, L. (1993). Estructura trófica de las comunidades de insectos en nuevos medios acuáticos. Anales de Biología, 19, 7-18.

Watson, J. (1982). A truly terrestrial dragonfly larva from Australia (Odonata: Corduliidae). Journal of Australian Entomology Society, 21, 309-311.

Westfall, M., \& May, M. (1996). Damselflies of North America. Gainesville, Florida: Scientific Publisher.

Whittaker, R. (1965). Dominance and diversity in land plant communities. Science, 147, 250-260. 